\title{
On Upper $\boldsymbol{k}$-Record Values from the Generalized Linear Exponential Distribution
}

\author{
M. Alam, M. A. Khan ${ }^{*}$, R. U. Khan \\ Department of Statistics and Operations Research, Aligarh Muslim University, Aligarh, 202 002, India
}

\section{ARTICLE INFO}

Article History

Received 12 Jun 2020

Accepted 18 Jan 2021

\section{Keywords}

Record values

Single moments

Product moments

Recurrence relations

Characterization

\section{ABSTRACT}

In this paper, we derive the exact expressions as well as recurrence relations for single and product moment of generalized upper record values from the four-parameter generalized linear exponential distribution. Further, we characterize the given distribution through conditional expectation and recurrence relations.

(c) 2021 The Authors. Published by Atlantis Press B.V. This is an open access article distributed under the CC BY-NC 4.0 license (http://creativecommons.org/licenses/by-nc/4.0/).

\section{INTRODUCTION}

The exponential, Rayleigh, Weibull, and the linear exponential distribution (LED) are widely used to model lifetime data. These distribution have several desirable properties which are frequently used in analyzing lifetime data. In particular, the generalized linear exponential distribution (GLED) having linearly increasing failure rates is very useful for modeling lifetime data. The exponential and Rayleigh distributions are special cases of LED. Since the LED does not provide a reasonable parametric fit for bathtub shape (decreasing, nonlinear increasing, nonmonotone failure rates). Therefore, a lot of work is devoted to GLED and propose some new distribution which are more flexible. So, in this continuation Mahmoud and Alam [1] introduced the four-parameter GLED, which defines the different distributional properties of GLED. Lee and Tsai [2] modify the moment of the GLED in a different way, which is more robust. The GLED shows the increasing, decreasing, and bathtub-shaped hazard rate functions which are quite useful in reliability and biological studies.

A random variable $X$ is said to have a GLED with four parameters $\theta, \alpha>0$ and $\beta, \lambda \geq 0$, if its probability density function ( $p d f)$ is of the form

$$
f(x)=\alpha(\beta+\theta x)\left(\frac{\theta}{2} x^{2}+\beta x-\lambda\right)^{\alpha-1} \exp \left\{-\left(\frac{\theta}{2} x^{2}+\beta x-\lambda\right)^{\alpha}\right\} I_{(\Psi, \infty)}(x), \quad x>0,
$$

where

$$
I_{(\Psi, \infty)}(x)= \begin{cases}1 & x>\Psi \\ 0 & \text { otherwise }\end{cases}
$$

is the indicator function, and

$$
\Psi=\frac{-\beta+\sqrt{\beta^{2}+2 \theta \lambda}}{\theta}
$$

and the distribution function $(d f)$ is

$$
F(x)=\left[1-\exp \left\{-\left(\frac{\theta}{2} x^{2}+\beta x-\lambda\right)^{\alpha}\right\}\right] I_{(\Psi, \infty)}(x), \quad x>0 .
$$

\footnotetext{
*Corresponding author. Email: khanazam2808@gmail.com
} 
It is easy to see that

$$
\left(\frac{\theta}{2} x^{2}+\beta x-\lambda\right) f(x)=\alpha(\beta+\theta x)[-\ln \bar{F}(x)][\bar{F}(x)]
$$

where

$$
\bar{F}(x)=1-F(x) .
$$

The concept of record values was introduced by Chandler [3]. An observation is called a record if its value is greater (or less) than all the previous observations. Record values are used in a wide variety of practical situations, such as industrial stress testing, meteorological analysis, hydrology, seismology, oil, mining surveys, sports, and athletic events. For a survey on important results in this area one may refer to Ahsanullah [4], Kamps [5], Arnold et al. [6] and Ahsanullah and Nevzorov [7]. Dziubdziela and Kopcoiński [8] have generalized the concept of record values of Chandler [3] by random variables of a more generalized nature and called them the $k$-th record values. Later, Minimol and Thomas ([9], p. 487) called the record values defined by Dziubdziela and Kopcoiński [8] also as the generalized record values, since the $r$-th member of the sequence of the classical record values is also known as the $r$-th record value. By setting $k=1$, we obtain ordinary record statistics.

Let $\left\{X_{n}, n \geq 1\right\}$ be a sequence of independent and identical distributed (iid) continuous random variables with $d f F(x)$ and $p d f f(x)$. Then for a fixed positive integer $k \geq 1$, the sequence of $k$-th upper record times $\left\{U_{n}^{(k)}, n \geq 1\right\}$ is defined as Nevzorov [10]:

$$
U_{1}^{(k)}=k
$$

and for $n \geq 1$,

$$
U_{n+1}^{(k)}=\min \left\{j: j>U_{n}^{(k)}, X_{j}>X_{U_{n}^{(k)}-k+1: U_{n}^{(k)}}\right\}
$$

The sequence $\left\{Y_{n}^{(k)}, n \geq 1\right\}$, where $Y_{n}^{(k)}=X_{U_{n}^{(k)}: U_{n}^{(k)}+k-1}$ is called the sequence of generalized upper record values ( $k$-th upper record values) of $\left\{X_{n}, n \geq 1\right\}$. Note that for $k=1$, we have $Y_{n}^{(1)}=X_{U_{n}}, n \geq 1$, which are the record values of $\left\{X_{n}, n \geq 1\right\}$ as defined in Ahsanullah [4]. The $p d f$ of $Y_{n}^{(k)}$ and the joint $p d f$ of $Y_{m}^{(k)}$ and $Y_{n}^{(k)}$ are given by (Dziubdziela and Kopcoiński [8], Grudzień [11])

$$
\begin{aligned}
& f_{Y_{n}^{(k)}}(x)=\frac{k^{n}}{(n-1) !}[-\ln \bar{F}(x)]^{n-1}[\bar{F}(x)]^{k-1} f(x), \quad n \geq 1, \\
& f_{Y_{m}^{(k)}, Y_{n}^{(k)}}(x, y)= \frac{k^{n}}{(m-1) !(n-m-1) !}[-\ln \bar{F}(x)]^{m-1} \frac{f(x)}{\bar{F}(x)} \\
& \times[\ln \bar{F}(x)-\ln \bar{F}(y)]^{n-m-1}[\bar{F}(y)]^{k-1} f(y), \quad x<y, 1 \leq m<n,
\end{aligned}
$$

and the conditional $p d f$ of $Y_{n}^{(k)}$ given $Y_{m}^{(k)}=x$, is

$$
f_{Y_{n}^{(k)} \mid Y_{m}^{(k)}}(y \mid x)=\frac{k^{n-m}}{(n-m-1) !}[\ln \bar{F}(x)-\ln \bar{F}(y)]^{n-m-1}\left[\frac{\bar{F}(y)}{\bar{F}(x)}\right]^{k-1} \frac{f(y)}{\bar{F}(x)}, \quad x<y
$$

For some recent developments on generalized record values with special reference to those arising from exponential, Gumbel, Pareto, generalized Pareto, Burr, Weibull, Gompertz, Makeham, modified-Weibull, exponential-Weibull, additive Weibull, and Kumaraswamy loglogistic distributions, see Grudzień and Szynal [12,13], Pawlas and Szynal [14-1516], Minimol and Thomas [9,17], Khan and Khan [18], Khan et al. [19,20], and Singh et al. [21], respectively. In this paper, we mainly focus on the study of generalized upper record values arising from the GLED and discussed exact explicit expressions as well as several recurrence relations satisfied by single and product moments. In addition, conditional expectations and recurrence relations of generalized record values are used to characterize this distribution. 


\section{RELATIONS FOR SINGLE MOMENTS}

In this section, we derive the exact expressions for single moments of generalized upper record values and recurrence relations in the following theorems:

Theorem 2.1. For the distribution given in (1.1). Fix a positive integer $k \geq 1$, for $n \geq 1,\left(\beta^{2}+2 \theta \lambda\right)>0$ and $j=0,1, \ldots$

$$
\begin{aligned}
E\left(Y_{n}^{(k)}\right)^{j}= & \frac{1}{(n-1) !}\left[\sum_{u=0}^{j} \sum_{v=0}^{\infty}\left(\begin{array}{c}
j \\
u
\end{array}\right)\left(\begin{array}{c}
\frac{j-u}{2} \\
v
\end{array}\right)(-\beta)^{u} 2^{v} \frac{\left(\beta^{2}+2 \theta \lambda\right)^{\frac{j-u}{2}-v}}{\theta^{j-v} k^{\frac{v}{\alpha}}} \gamma\left\{\frac{v}{\alpha}+n, k\left(\frac{\beta^{2}+2 \theta \lambda}{2 \theta}\right)^{\alpha}\right\}\right. \\
& +\sum_{u=0}^{j} \sum_{v=0}^{\infty}\left(\begin{array}{c}
j \\
u
\end{array}\right)\left(\begin{array}{c}
\frac{j-u}{2} \\
v
\end{array}\right)(-\beta)^{u} 2^{\frac{j-u}{2}-v} \frac{\left(\beta^{2}+2 \theta \lambda\right)^{v}}{\theta^{\frac{j+u}{2}+v} k^{\frac{j-u}{2 \alpha}-\frac{v}{\alpha}}} \Gamma\left\{\frac{j-u}{2 \alpha}-\frac{v}{\alpha}+n, k\left(\frac{\beta^{2}+2 \theta \lambda}{2 \theta}\right)^{\alpha}\right\},
\end{aligned}
$$

where $\Gamma(s, t)=\int_{t}^{\infty} x^{s-1} e^{-x} d x$ and $\gamma(s, t)=\int_{0}^{t} x^{s-1} e^{-x} d x$ are the upper and lower incomplete gamma functions, respectively.

Proof. In the view of (1.4), we have

$$
E\left(Y_{n}^{(k)}\right)^{j}=\frac{k^{n}}{(n-1) !} \int_{-\infty}^{\infty} x^{j}[-\ln \bar{F}(x)]^{n-1}[\bar{F}(x)]^{k-1} f(x) d x, n \geq 1
$$

Now using (1.3), we get

$$
\begin{aligned}
E\left(Y_{n}^{(k)}\right)^{j} & =\frac{\alpha k^{n}}{(n-1) !} \int_{\infty}^{\Psi} \frac{x^{j}(\beta+\theta x)}{\left(\frac{\theta}{2} x^{2}+\beta x-\lambda\right)}[-\ln \bar{F}(x)]^{n}[\bar{F}(x)]^{k} d x \\
& =\frac{\alpha k^{n}}{(n-1) !} \int_{\infty}^{\Psi} \frac{x^{j}(\beta+\theta x)}{\left(\frac{\theta}{2} x^{2}+\beta x-\lambda\right)}\left[\left(\frac{\theta}{2} x^{2}+\beta x-\lambda\right)^{\alpha}\right]^{n}\left[e^{-\left(\frac{\theta}{2} x^{2}+\beta x-\lambda\right)^{\alpha}}\right]^{k} d x,
\end{aligned}
$$

after substituting $t=\left(\frac{\theta}{2} x^{2}+\beta x-\lambda\right)^{\alpha}$ in (2.2), we get

$$
E\left(Y_{n}^{(k)}\right)^{j}=\frac{k^{n}}{(n-1) !} \int_{0}^{\infty}\left[\frac{-\beta+\sqrt{2 \theta t^{1 / \alpha}+\left(\beta^{2}+2 \theta \lambda\right)}}{\theta}\right]^{j} t^{n-1} e^{-k t} d t
$$

On using binomial expansion in (2.3) and we have

$$
\begin{aligned}
E\left(Y_{n}^{(k)}\right)^{j} & =\frac{k^{n}}{(n-1) !} \sum_{u=0}^{j}\left(\begin{array}{c}
j \\
u
\end{array}\right) \frac{(-\beta)^{u}}{\theta^{j}} \int_{\infty}^{0}\left[2 \theta t^{1 / \alpha}+\left(\beta^{2}+2 \theta \lambda\right)\right]^{\frac{j-u}{2}} t^{n-1} e^{-k t} d t . \\
& =\frac{k^{n}}{(n-1) !} \sum_{u=0}^{j}\left(\begin{array}{c}
j \\
u
\end{array}\right) \frac{(-\beta)^{u}}{\theta^{j}} \int_{\infty}^{0}\left[1+\frac{\beta^{2}+2 \theta \lambda}{2 \theta t^{1 / \alpha}}\right]^{\frac{j-u}{2}}\left(2 \theta t^{1 / \alpha}\right)^{\frac{j-u}{2}} t^{n-1} e^{-k t} d t . \\
& =\frac{k^{n}}{(n-1) !} \sum_{u=0}^{j}\left(\begin{array}{l}
j \\
u
\end{array}\right) \frac{(-\beta)^{u}}{\theta^{j}} \int_{\infty}^{0}\left[1+\frac{1}{\psi t^{1 / \alpha}}\right]^{\frac{j-u}{2}}\left(2 \theta t^{1 / \alpha}\right)^{\frac{j-u}{2}} t^{n-1} e^{-k t} d t .
\end{aligned}
$$


where $\psi=\frac{2 \theta}{\beta^{2}+2 \theta \lambda}$, by the expression $\left|\frac{\beta^{2}+2 \theta \lambda}{2 \theta t^{1 / \alpha}}\right|<1$ only if $\left(\frac{\beta^{2}+2 \theta \lambda}{2 \theta}\right)^{\alpha}<t<\infty$. We can verify that $\left|\psi t^{1 / \alpha}\right|<1$ if $0<t<\left(\frac{1}{\psi}\right)^{\alpha}$ and $\left|\frac{1}{\psi t^{1 / \alpha}}\right|<1$ if $\left(\frac{1}{\psi}\right)^{\alpha}<t<\infty$, thus the integral in (2.4) should be separated into two parts as:

$$
\begin{aligned}
E\left(Y_{n}^{(k)}\right)^{j}= & \frac{k^{n}}{(n-1) !} \sum_{u=0}^{j}\left(\begin{array}{c}
j \\
u
\end{array}\right) \frac{(-\beta)^{u}}{\theta^{j}}\left[\int_{0}^{\left(\frac{1}{\psi}\right)^{\alpha}}\left(1+\frac{1}{\psi t^{1 / \alpha}}\right)^{\frac{j-u}{2}}\left(2 \theta t^{1 / \alpha}\right)^{\frac{j-u}{2}}\right. \\
& \left.\times t^{n-1} e^{-k t} d t+\int_{\left(\frac{1}{\psi}\right)^{\alpha}}^{\infty}\left[1+\frac{1}{\psi t^{1 / \alpha}}\right]^{\frac{j-u}{2}}\left(2 \theta t^{1 / \alpha}\right)^{\frac{j-u}{2}} t^{n-1} e^{-k t} d t\right] \\
= & \frac{k^{n}}{(n-1) !} \sum_{u=0}^{j}\left(\begin{array}{c}
j \\
u
\end{array}\right) \frac{(-\beta)^{u}}{\theta^{j}}\left(\beta^{2}+2 \theta \lambda\right)^{\frac{j-u}{2}}\left[\int_{0}^{\left(\frac{1}{\psi}\right)^{\alpha}}\left[1+\psi t^{1 / \alpha}\right]^{\frac{j-u}{2}}\right. \\
& \left.\times t^{n-1} e^{-k t} d t+\int_{\left(\frac{1}{\psi}\right)^{\alpha}}^{\infty}\left[1+\frac{1}{\psi t^{1 / \alpha}}\right]^{\frac{j-u}{2}}\left(\psi t^{1 / \alpha}\right)^{\frac{j-u}{2}} t^{n-1} e^{-k t} d t\right] .
\end{aligned}
$$

On using the binomial expansion in (2.5), we get:

$$
\begin{aligned}
E\left(Y_{n}^{(k)}\right)^{j}= & \frac{k^{n}}{(n-1) !} \sum_{u=0}^{j}\left(\begin{array}{c}
j \\
u
\end{array}\right) \frac{(-\beta)^{u}}{\theta^{j}}\left(\beta^{2}+2 \theta \lambda\right)^{\frac{j-u}{2}}\left[\sum_{v=0}^{\infty}\left(\begin{array}{c}
\frac{j-u}{2} \\
v
\end{array}\right) \psi^{v} \times \int_{0}^{\left(\frac{1}{\psi}\right)^{\alpha}} t^{\frac{v}{\alpha}+n-1} e^{-k t} d t\right. \\
& \left.+\sum_{v=0}^{\infty}\left(\begin{array}{c}
\frac{j-u}{2} \\
v
\end{array}\right) \psi^{\frac{j-u}{2}-v} \int_{\infty}^{\left(\frac{1}{\psi}\right)^{\alpha}} t^{\frac{j-u}{2 \alpha}-\frac{v}{\alpha}+n-1} e^{-k t} d t\right] . \\
= & \frac{k^{n}}{(n-1) !} \sum_{u=0}^{j}\left(\begin{array}{c}
j \\
u
\end{array}\right) \frac{(-\beta)^{u}}{\theta^{j}}\left(\beta^{2}+2 \theta \lambda\right)^{\frac{j-u}{2}}\left[\sum_{v=0}^{\infty}\left(\begin{array}{c}
\frac{j-u}{2} \\
v
\end{array}\right)\left(\frac{2 \theta}{\beta^{2}+2 \theta \lambda}\right)^{v} \times \int_{0}^{\left(\frac{\beta^{2}+2 \theta \lambda}{2 \theta}\right)^{\alpha}} t^{\frac{v}{\alpha}+n-1} e^{-k t} d t\right. \\
& \left.+\sum_{v=0}^{\infty}\left(\begin{array}{c}
\frac{j-u}{2} \\
v
\end{array}\right)\left(\frac{2 \theta}{\beta^{2}+2 \theta \lambda}\right)^{\frac{j-u}{2}-v} \int_{\infty}^{\left(\frac{\beta^{2}+2 \theta \lambda}{2 \theta}\right)^{\alpha}} \frac{j-u}{t^{\frac{j \alpha}{2 \alpha}}-\frac{v}{\alpha}+n-1} e^{-k t} d t\right],
\end{aligned}
$$

using the incomplete gamma function in (2.6), we get the result as given in (2.1).

Corollary 2.1. The $j$-th moment of the $k$-th record values from GLED has another form:

$$
\begin{aligned}
E\left(Y_{n}^{(k)}\right)^{j}= & \frac{1}{(n-1) !}\left[\frac{\left(\beta^{2}+2 \theta \lambda\right)^{\frac{j}{2}}}{\theta^{j}} \gamma\left\{n, k\left(\frac{\beta^{2}+2 \theta \lambda}{2 \theta}\right)^{\alpha}\right\}+\frac{2^{\frac{j}{2}}}{\theta^{\frac{j}{2}} \frac{j}{2 \alpha}} \Gamma\left\{\frac{j}{2 \alpha}+n, k\left(\frac{\beta^{2}+2 \theta \lambda}{2 \theta}\right)^{\alpha}\right\}\right. \\
& +\sum_{u=1}^{j} \sum_{v=1}^{\infty}\left(\begin{array}{c}
j \\
u
\end{array}\right)\left(\begin{array}{c}
\frac{j-u}{2} \\
v
\end{array}\right)(-\beta)^{u} 2^{v} \frac{\left(\beta^{2}+2 \theta \lambda\right)^{\frac{j-u}{2}-v}}{\theta^{j-v} k \frac{v}{\alpha}} \gamma\left\{\frac{v}{\alpha}+n, k\left(\frac{\beta^{2}+2 \theta \lambda}{2 \theta}\right)^{\alpha}\right\} \\
& \left.+\sum_{u=1}^{j} \sum_{v=1}^{\infty}\left(\begin{array}{c}
j \\
u
\end{array}\right)\left(\begin{array}{c}
\frac{j-u}{2} \\
v
\end{array}\right)(-\beta)^{u} 2 \frac{j-u}{2}-v \frac{\left(\beta^{2}+2 \theta \lambda\right)^{v}}{\theta^{\frac{j+u}{2}+v} k \frac{j-u}{2 \alpha}-\frac{v}{\alpha}} \Gamma\left\{\frac{j-u}{2 \alpha}-\frac{v}{\alpha}+n, k\left(\frac{\beta^{2}+2 \theta \lambda}{2 \theta}\right)^{\alpha}\right\}\right] .
\end{aligned}
$$

Proof. On expanding (2.1), at $u=0$ and $v=0$. Hence, the result. 


\section{Remark 2.1.}

i) Setting $j=0$ in $(2.1)$, then

$$
R H S=\frac{1}{(n-1) !}\left[\gamma\left\{n, k\left(\frac{\beta^{2}+2 \theta \lambda}{2 \theta}\right)^{\alpha}\right\}+\Gamma\left\{n, k\left(\frac{\beta^{2}+2 \theta \lambda}{2 \theta}\right)^{\alpha}\right\}\right]
$$

by using the formula $[\Gamma(s, t)+\gamma(s, t)]=\Gamma(s)=\int_{0}^{\infty} t^{s-1} e^{-t} d t$, we get

$$
R H S=\frac{1}{(n-1) !} \Gamma(n)=1=L H S \quad \text { as } \quad \Gamma(n)=(n-1) !
$$

ii) Setting $k=1$ and $n=1$ in (2.7), we get the exact expression for the $j$-th ordinary moment of GLED as obtained by Lee and Tsai [2].

iii) Setting $\beta=\lambda=0$ in (2.7), we get the exact expression for single moments of generalized upper records from Weibull distribution

$$
E\left(Y_{n}^{(k)}\right)^{j}=\frac{1}{(n-1) !}\left[\frac{2^{\frac{j}{2}}}{\theta^{\frac{j}{2}} k^{\frac{j}{2 \alpha}}} \Gamma\left\{\frac{j}{2 \alpha}+n, 0\right\}\right]=\left(\sqrt{\frac{2}{k^{1 / \alpha} \theta}}\right)^{j} \frac{\Gamma\left(\frac{j}{2 \alpha}+n\right)}{(n-1) !}
$$

as obtained by Kamps [5] and at $k=1$, we get the result as given in Nagaraja [22].

iv) Setting $\alpha=\frac{1}{2}$ in (2.8), we get the exact expression for single moments of generalized upper records from the exponential distribution

$$
E\left(Y_{n}^{(k)}\right)^{j}=\frac{1}{\left(k \sqrt{\frac{\theta}{2}}\right)^{j}} \frac{\Gamma(j+n)}{(n-1) !}
$$

as obtained by Kamps [5] and at $k=1$ in Ahsanullah [23].

v) Setting $\alpha=1$ in (2.8), we get the exact expression for single moments of generalized upper records from Rayleigh distribution

$$
E\left(Y_{n}^{(k)}\right)^{j}=\left(\sqrt{\frac{2}{k \theta}}\right)^{j} \frac{\Gamma\left(\frac{j}{2}+n\right)}{(n-1) !}
$$

at $k=1$ we get the similar result as given in Ahsanullah and Shakil [24].

vi) Setting $\lambda=0$ and $\alpha=1$ in (2.1), we get the exact expression for single moments of generalized upper records from the LED

$$
\begin{aligned}
E\left(Y_{n}^{(k)}\right)^{j}= & \frac{1}{(n-1) !}\left[\sum_{u=0}^{j} \sum_{v=0}^{\infty}\left(\begin{array}{c}
j \\
u
\end{array}\right)\left(\begin{array}{c}
\frac{j-u}{2} \\
v
\end{array}\right)(-1)^{u} 2^{v} \frac{\beta^{j-2 v}}{\theta^{j-v} k^{v}} \gamma\left\{v+n, k\left(\frac{\beta^{2}}{2 \theta}\right)\right\}\right. \\
& \left.+\sum_{u=0}^{j} \sum_{v=0}^{\infty}\left(\begin{array}{c}
j \\
u
\end{array}\right)\left(\begin{array}{c}
\frac{j-u}{2} \\
v
\end{array}\right)(-1)^{u} 2^{\frac{j-u}{2}-v} \frac{\beta^{u+2 v}}{\theta^{\frac{j+u}{2}+v} \frac{j-u}{2}-v} \Gamma\left\{\frac{j-u}{2}-v+n, k\left(\frac{\beta^{2}}{2 \theta}\right)\right\}\right] .
\end{aligned}
$$

vii) Setting $\beta=0$ in (2.7), we get the exact expression for single moments of generalized upper records from the generalized Rayleigh distribution

$$
\begin{aligned}
E\left(Y_{n}^{(k)}\right)^{j} & =\frac{1}{(n-1) !}\left[\frac{(2 \theta \lambda)^{\frac{j}{2}}}{\theta^{j}} \gamma\left\{n, k \lambda^{\alpha}\right\}+\frac{2^{\frac{j}{2}}}{\theta^{\frac{j}{2}} \frac{j}{k^{2 \alpha}}} \Gamma\left\{\frac{j}{2 \alpha}+n, k \lambda^{\alpha}\right\}\right] \\
& =\left(\sqrt{\frac{2}{\theta}}\right)^{j} \frac{1}{(n-1) !}\left[\lambda^{\frac{j}{2}} \gamma\left\{n, k \lambda^{\alpha}\right\}+\frac{1}{\frac{j}{2 \alpha}} \Gamma\left\{\frac{j}{2 \alpha}+n, k \lambda^{\alpha}\right\}\right] .
\end{aligned}
$$


Theorem 2.2. For the distribution given in (1.1), fix a positive integer $k \geq 1$, for $n \geq 1$ and $j=0,1, \ldots$

$$
\begin{aligned}
E\left(Y_{n}^{(k)}\right)^{j}= & \frac{n \alpha \theta}{(j+2) \lambda}\left[\left(1+\frac{j+2}{2 n \alpha}\right) E\left(Y_{n}^{(k)}\right)^{j+2}-E\left(Y_{n+1}^{(k)}\right)^{j+2}\right] \\
& +\frac{n \alpha \beta}{(j+1) \lambda}\left[\left(1+\frac{j+1}{n \alpha}\right) E\left(Y_{n}^{(k)}\right)^{j+1}-E\left(Y_{n+1}^{(k)}\right)^{j+1}\right] .
\end{aligned}
$$

Proof. From (1.4) for $n \geq 1$ and $j=0,1, \ldots$, we have

$$
\frac{\theta}{2}\left[E\left(Y_{n}^{(k)}\right)^{j+2}\right]+\beta\left[E\left(Y_{n}^{(k)}\right)^{j+1}\right]-\lambda\left[E\left(Y_{n}^{(k)}\right)^{j}\right]=\frac{k^{n}}{(n-1) !} \times \int_{\Psi}^{\infty}\left(\frac{\theta}{2} x^{2}+\beta x-\lambda\right) x^{j}[-\ln \bar{F}(x)]^{n-1}(\bar{F}(x))^{k-1} f(x) d x
$$

on using (1.3) in (2.10), we get

$$
\begin{gathered}
\frac{\theta}{2}\left[E\left(Y_{n}^{(k)}\right)^{j+2}\right]+\beta\left[E\left(Y_{n}^{(k)}\right)^{j+1}\right]-\lambda\left[E\left(Y_{n}^{(k)}\right)^{j}\right] \\
=\frac{k^{n}}{(n-1) !} \int_{\Psi}^{\infty} \alpha(\beta+\theta x) x^{j}[-\ln \bar{F}(x)]^{n}[\bar{F}(x)]^{k} d x \\
=\frac{\alpha \beta k^{n}}{(n-1) !} \int_{\Psi}^{\infty} x^{j}[-\ln \bar{F}(x)]^{n}[\bar{F}(x)]^{k} d x+\frac{\alpha \theta k^{n}}{(n-1) !} \int_{\Psi}^{\infty} x^{j+1}[-\ln \bar{F}(x)]^{n}[\bar{F}(x)]^{k} d x .
\end{gathered}
$$

Now applying Lemma 2.1 stated in Khan et al. [19] to (2.11), we have

$$
\begin{gathered}
\frac{\theta}{2}\left[E\left(Y_{n}^{(k)}\right)^{j+2}\right]+\beta\left[E\left(Y_{n}^{(k)}\right)^{j+1}\right]-\lambda\left[E\left(Y_{n}^{(k)}\right)^{j}\right] \\
=\frac{n \alpha \theta}{j+2}\left[E\left(Y_{n+1}^{(k)}\right)^{j+2}-E\left(Y_{n}^{(k)}\right)^{j+2}\right]+\frac{n \alpha \beta}{j+1}\left[E\left(Y_{n+1}^{(k)}\right)^{j+1}-E\left(Y_{n}^{(k)}\right)^{j+1}\right],
\end{gathered}
$$

after arranging (2.12) we get the yields given in (2.9).

Remark 2.2. If we put

i) $\quad \beta=\lambda=0$ in (2.11) we get the recurrence relations for single moment of generalized upper records from Weibull distribution

$$
E\left(Y_{n+1}^{(k)}\right)^{j+2}=\left(1+\frac{j+2}{2 n \alpha}\right) E\left(Y_{n}^{(k)}\right)^{j+2}
$$

at $j^{\prime}=j+2$, we get similar result as obtained by Khan and Khan [18].

ii) $\quad \beta=\lambda=0$ and $\alpha=\frac{1}{2}$ in (2.11) we get the recurrence relations for single moment of generalized upper records from the exponential distribution

$$
E\left(Y_{n+1}^{(k)}\right)^{j+2}=\left(1+\frac{j+2}{n}\right) E\left(Y_{n}^{(k)}\right)^{j+2}
$$

at $j^{\prime}=j+2$, we get similar result as obtained by Khan and Khan [18].

iii) $\beta=\lambda=0$ and $\alpha=1$ in (2.11) we get the recurrence relations for single moment of generalized upper records from Rayleigh distribution

$$
E\left(Y_{n+1}^{(k)}\right)^{j+2}=\left(1+\frac{j+2}{2 n}\right) E\left(Y_{n}^{(k)}\right)^{j+2}
$$

at $j^{\prime}=j+2$, we get similar result as obtained by Khan and Khan [18]. 
iv) $\lambda=0$ and $\alpha=1$ in (2.11) we get the recurrence relations for single moment of generalized upper records from the LED

$$
E\left(Y_{n+1}^{(k)}\right)^{j+2}=\left(1+\frac{j+2}{2 n}\right) E\left(Y_{n}^{(k)}\right)^{j+2}+\frac{(j+2) \beta}{(j+1) \theta}\left[\left(1+\frac{j+1}{n}\right) E\left(Y_{n}^{(k)}\right)^{j+1}-E\left(Y_{n+1}^{(k)}\right)^{j+1}\right]
$$

v) $\beta=0$ in (2.8) we get the recurrence relations for single moment of generalized upper records from the generalized Rayleigh distribution

$$
E\left(Y_{n}^{(k)}\right)^{j}=\frac{n \alpha \theta}{(j+2) \lambda}\left[\left(1+\frac{j+2}{2 n \alpha}\right) E\left(Y_{n}^{(k)}\right)^{j+2}-E\left(Y_{n+1}^{(k)}\right)^{j+2}\right]
$$

Corollary 2.2. The recurrence relation for single moments of the upper record values from GLED has the form

$$
\begin{aligned}
\lambda E\left(X_{U_{n}}\right)^{j}= & \frac{n \alpha \theta}{j+2}\left[\left(1+\frac{j+2}{2 n \alpha}\right) E\left(X_{U_{n}}\right)^{j+2}-E\left(X_{U_{n+1}}\right)^{j+2}\right] \\
& +\frac{n \alpha \beta}{j+1}\left[\left(1+\frac{j+1}{n \alpha}\right) E\left(X_{U_{n}}\right)^{j+1}-E\left(X_{U_{n+1}}\right)^{j+1}\right] .
\end{aligned}
$$

\section{Remark 2.3. If we put}

i) $\beta=\lambda=0$ in (2.12), we get the recurrence relations for single moment of upper records from Weibull distribution, which is similar to the result as obtained by Balakrishnan and Chan [25] for $j^{\prime}=j+2$.

ii) $\quad \beta=\lambda=0$ and $\alpha=\frac{1}{2}$ and (2.12) in, the results for the single moments of upper records obtained by Balakrishnan and Chan [25] at $j^{\prime}=j+2$ for the exponential distribution is deduced.

iii) $\beta=\lambda=0$ and $\alpha=1$ in (2.12), the results for the single moments of upper records obtained by Balakrishnan and Chan [25] at $j^{\prime}=j+2$ for Rayleigh distribution is deduced.

iv) $\lambda=0$ and $\alpha=1$ in (2.12), we get the recurrence relations for single moments of upper records from the LED

$$
E\left(X_{U_{n+1}}\right)^{j+2}=\left(1+\frac{j+2}{2 n}\right) E\left(X_{U_{n}}\right)^{j+2}+\frac{(j+2) \beta}{(j+\mid 1) \theta}\left[\left(1+\frac{j+1}{n}\right) E\left(X_{U_{n}}\right)^{j+1}-E\left(X_{U_{n+1}}\right)^{j+1}\right]
$$

v) Setting $\beta=0$ in (2.12), we get the recurrence relations for single moments of upper records from the generalized Rayleigh distribution

\begin{tabular}{|c|c|c|c|c|c|c|}
\hline \multirow[b]{3}{*}{$n$} & \multicolumn{3}{|c|}{$E(X)$} & \multicolumn{3}{|c|}{$E(X)^{2}$} \\
\hline & \multicolumn{3}{|c|}{$\alpha=0.5, \theta=1, \beta=2$} & \multicolumn{3}{|c|}{$\alpha=0.5, \theta=1, \beta=2$} \\
\hline & $k=1$ & $k=2$ & $k=3$ & $k=1$ & $k=2$ & $\mathbf{k}=\mathbf{3}$ \\
\hline 1 & 0.736241 & 0.2210499 & 0.1040861 & 2.110072 & 0.2316011 & 0.0561998 \\
\hline 2 & 1.947826 & 0.6230462 & 0.3010611 & 8.417389 & 1.015631 & 0.2581783 \\
\hline 3 & 3.449258 & 1.16617 & 0.5780411 & 20.40594 & 2.670637 & 0.7090046 \\
\hline 4 & 5.12462 & 1.816237 & 0.9222053 & 39.00304 & 5.470104 & 1.511246 \\
\hline 5 & 6.905298 & 2.546131 & 1.321817 & 64.75762 & 9.630955 & 2.758801 \\
\hline 6 & 8.751285 & 3.335303 & 1.766684 & 97.98972 & 15.31757 & 4.533193 \\
\hline 7 & 10.63908 & 4.168577 & 2.248265 & 138.8874 & 22.65138 & 6.90277 \\
\hline 8 & 12.55456 & 5.03488 & 2.759559 & 187.5635 & 31.72096 & 9.923529 \\
\hline 9 & 14.48904 & 5.926162 & 3.294916 & 244.0877 & 42.59071 & 13.64067 \\
\hline 10 & 16.43696 & 6.836557 & 3.849815 & 308.5043 & 55.30755 & 18.09037 \\
\hline
\end{tabular}

$$
E\left(X_{U_{n}}\right)^{j}=\frac{n \alpha \theta}{(j+2) \lambda}\left[\left(1+\frac{j+2}{2 n \alpha}\right) E\left(X_{U_{n}}\right)^{j+2}-E\left(X_{U_{n+1}}\right)^{j+2}\right]
$$

Numerical computations for moments of $k$-th upper record values from the GLED for arbitrary chosen values of $\alpha, \beta, \theta$. Setting $\lambda=0$ without loss of generality and various sample size $n=1,2, \ldots 10$ are given in Tables 1 and 2 .

Table 1 Moments of the generalized linear exponential distribution(GLED). 
Table 2 Moments of the generalized linear exponential distribution(GLED).

\begin{tabular}{|c|c|c|c|c|c|c|}
\hline \multirow[b]{3}{*}{$n$} & \multicolumn{3}{|c|}{$E(X)^{3}$} & \multicolumn{3}{|c|}{$E(X)^{4}$} \\
\hline & \multicolumn{3}{|c|}{$\alpha=0.5, \theta=1, \beta=2$} & \multicolumn{3}{|c|}{$\alpha=0.5, \theta=1, \beta=2$} \\
\hline & $k=1$ & $k=2$ & $k=3$ & $k=1$ & $k=2$ & $k=3$ \\
\hline 1 & 10.71348 & 0.479532 & 0.064216 & 77.53965 & 1.505019 & 0.116498 \\
\hline 2 & 55.65176 & 2.772377 & 0.393788 & 490.8589 & 10.64161 & 0.879686 \\
\hline 3 & 168.2068 & 9.18847 & 1.377652 & 1762.711 & 42.06369 & 3.692394 \\
\hline 4 & 388.0785 & 22.94524 & 3.613889 & 4734.55 & 122.7896 & 11.38394 \\
\hline 5 & 758.6281 & 48.00968 & 7.906454 & 10597.46 & 295.4639 & 28.7853 \\
\hline 6 & 1325.249 & 88.92437 & 15.24622 & 20908.68 & 620.8854 & 63.26942 \\
\hline 7 & 2134.446 & 150.654 & 26.78464 & 37600.07 & 1179.848 & 125.224 \\
\hline 8 & 3233.338 & 238.4644 & 43.80586 & 62982.53 & 2074.428 & 228.4448 \\
\hline 9 & 4669.385 & 357.8329 & 67.7008 & 99748.23 & 3428.868 & 390.451 \\
\hline 10 & 6490.247 & 514.3848 & 99.94468 & 150971.8 & 5390.161 & 632.7313 \\
\hline
\end{tabular}

\section{RELATIONS FOR PRODUCT MOMENTS}

In this section, we derived the recurrence relations for product moments of generalized upper record values. Before proving the main result, we shall prove the following lemma:

Lemma 3.1. Fix a positive integer $k \geq 1$, for $1 \leq m \leq n-2$ and $i, j=0,1, \ldots$,

$$
\begin{aligned}
E\left[\left(Y_{m+1}^{(k)}\right)^{i}\left(Y_{n}^{(k)}\right)^{j}\right]-E\left[\left(Y_{m}^{(k)}\right)^{i}\left(Y_{n}^{(k)}\right)^{j}\right]= & \frac{i k^{n}}{m !(n-m-1) !} \int_{-\infty}^{\infty} \int_{-\infty}^{y} x^{i-1} y^{j} \\
& \times[-\ln \bar{F}(x)]^{m}[\ln \bar{F}(x)-\ln \bar{F}(y)]^{n-m-1}[\bar{F}(y)]^{k-1} f(y) d x d y .
\end{aligned}
$$

Proof. From (1.5), we have

$$
\begin{aligned}
E\left[\left(Y_{m+1}^{(k)}\right)^{i}\left(Y_{n}^{(k)}\right)^{j}\right]-E\left[\left(Y_{m}^{(k)}\right)^{i}\left(Y_{n}^{(k)}\right)^{j}\right]= & \frac{k^{n}}{(m-1) !(n-m-1) !} \\
& \times \int_{-\infty-\infty}^{\infty} \int^{y} x^{i} y^{j}[-\ln \bar{F}(x)]^{m-1} \frac{f(x)}{\bar{F}(x)}[\ln \bar{F}(x)-\ln \bar{F}(y)]^{n-m-2} \\
& \times[\bar{F}(y)]^{k-1} f(y)\left\{[-\ln \bar{F}(x)] \frac{(n-m-1)}{m}-[\ln \bar{F}(x)-\ln \bar{F}(y)]\right\} d x d y
\end{aligned}
$$

Let

$$
\begin{gathered}
h(x, y)=\frac{1}{m}[\ln \bar{F}(x)-\ln \bar{F}(y)]^{n-m-1}[-\ln \bar{F}(x)]^{m} \\
\frac{\partial}{\partial x} h(x, y)=-[-\ln \bar{F}(x)]^{m-1} \frac{f(x)}{\bar{F}(x)}[\ln \bar{F}(x)-\ln \bar{F}(y)]^{n-m-2} \\
\times\left\{[-\ln \bar{F}(x)] \frac{(n-m-1)}{m}-[\ln \bar{F}(x)-\ln \bar{F}(y)]\right\} .
\end{gathered}
$$

On using the value of (3.4) in (3.2), we find that

$$
\begin{aligned}
E\left[\left(Y_{m+1}^{(k)}\right)^{i}\left(Y_{n}^{(k)}\right)^{j}\right]-E\left[\left(Y_{m}^{(k)}\right)^{i}\left(Y_{n}^{(k)}\right)^{j}\right]= & \frac{-k^{n}}{(m-1) !(n-m-1) !} \\
& \times \int_{-\infty}^{\infty} y^{j}[\bar{F}(y)]^{k-1} f(y)\left\{\int_{-\infty}^{y} x^{i} \frac{\partial}{\partial x} h(x, y) d x\right\} d y .
\end{aligned}
$$

Now, in view of (3.3)

$$
\int_{-\infty}^{y} x^{i} \frac{\partial}{\partial x} h(x, y) d x=-\frac{i}{m} \int_{-\infty}^{y} x^{i-1}[-\ln \bar{F}(x)]^{m}[\ln \bar{F}(x)-\ln \bar{F}(y)]^{n-m-1} d x .
$$


On substituting (3.6) in (3.5) and simplifying, we get the required result.

Theorem 3.1. For the distribution given in (1.1) and $k \geq 1, m \geq 1$ and $i, j=0,1, \ldots$

$$
\begin{aligned}
E\left[\left(Y_{m}^{(k)}\right)^{i}\left(Y_{m+1}^{(k)}\right)^{j}\right]= & \frac{m \alpha \theta}{(i+2) \lambda}\left[\left(1+\frac{i+2}{2 m \alpha}\right) E\left[\left(Y_{m}^{(k)}\right)^{i+2}\left(Y_{m+1}^{(k)}\right)^{j}\right]-E\left[\left(Y_{m+1}^{(k)}\right)^{i+j+2}\right]\right] \\
& +\frac{m \alpha \beta}{(i+1) \lambda}\left[\left(1+\frac{i+1}{m \alpha}\right) E\left[\left(Y_{m}^{(k)}\right)^{i+1}\left(Y_{m+1}^{(k)}\right)^{j}\right]-E\left[\left(Y_{m+1}^{(k)}\right)^{i+j+1}\right]\right]
\end{aligned}
$$

and for $1 \leq m \leq n-2$, and $i, j=0,1, \ldots$

$$
\begin{aligned}
E\left[\left(Y_{m}^{(k)}\right)^{i}\left(Y_{n}^{(k)}\right)^{j}\right]= & \frac{m \alpha \theta}{(i+2) \lambda}\left[\left(1+\frac{i+2}{2 m \alpha}\right) E\left[\left(Y_{m}^{(k)}\right)^{i+2}\left(Y_{n}^{(k)}\right)^{j}\right]-E\left[\left(Y_{m+1}^{(k)}\right)^{i+2}\left(Y_{n}^{(k)}\right)^{j}\right]\right] \\
& +\frac{m \alpha \beta}{(i+1) \lambda}\left[\left(1+\frac{i+1}{m \alpha}\right) E\left[\left(Y_{m}^{(k)}\right)^{i+1}\left(Y_{n}^{(k)}\right)^{j}\right]-E\left[\left(Y_{m+1}^{(k)}\right)^{i+1}\left(Y_{n}^{(k)}\right)^{j}\right]\right] .
\end{aligned}
$$

Proof. From (1.5), for $m \leq n-1$, we have

$$
\begin{aligned}
& \frac{\theta}{2} E\left[\left(Y_{m}^{(k)}\right)^{i+2}\left(Y_{n}^{(k)}\right)^{j}\right]+\beta E\left[\left(Y_{m}^{(k)}\right)^{i+1}\left(Y_{n}^{(k)}\right)^{j}\right]-\lambda E\left[\left(Y_{m}^{(k)}\right)^{i}\left(Y_{n}^{(k)}\right)^{j}\right] \\
= & \frac{k^{n}}{(m-1) !(n-m-1) !} \int_{\infty}^{\Psi} \int_{y}^{\Psi}\left(\frac{\theta}{2} x^{2}+\beta x-\lambda\right) x^{i} y^{j}[-\ln \bar{F}(x)]^{m-1} \frac{f(x)}{\bar{F}(x)} \\
& \times[\ln \bar{F}(x)-\ln \bar{F}(y)]^{n-m-1}[\bar{F}(y)]^{k-1} f(y) d x d y .
\end{aligned}
$$

In view of (1.3),

$$
\begin{aligned}
& \frac{\theta}{2} E\left[\left(Y_{m}^{(k)}\right)^{i+2}\left(Y_{n}^{(k)}\right)^{j}\right]+\beta E\left[\left(Y_{m}^{(k)}\right)^{i+1}\left(Y_{n}^{(k)}\right)^{j}\right]-\lambda E\left[\left(Y_{m}^{(k)}\right)^{i}\left(Y_{n}^{(k)}\right)^{j}\right] \\
= & \frac{\alpha k^{n}}{(m-1) !(n-m-1) !} \int_{\Psi}^{\infty} \int_{\Psi}^{y}(\beta+\theta x) x^{i} y^{j}[-\ln \bar{F}(x)]^{m} \\
& \times[\ln \bar{F}(x)-\ln \bar{F}(y)]^{n-m-1}[\bar{F}(y)]^{k-1} f(y) d x d y . \\
= & \frac{\alpha \beta k^{n}}{(m-1) !(n-m-1) !} \int_{\Psi}^{\infty} \int_{\Psi}^{y} x^{i} y^{j}[-\ln \bar{F}(x)]^{m}[\ln \bar{F}(x)-\ln \bar{F}(y)]^{n-m-1} \\
& \times[\bar{F}(y)]^{k-1} f(y) d x d y+\frac{\alpha \theta k^{n}}{(m-1) !(n-m-1) !} \int_{\Psi}^{\infty} \int_{\Psi}^{y} x^{i+1} y^{j}[-\ln \bar{F}(x)]^{m} \\
& \times[\ln \bar{F}(x)-\ln \bar{F}(y)]^{n-m-1}[\bar{F}(y)]^{k-1} f(y) d x d y .
\end{aligned}
$$

Using Lemma 3.1, we get

$$
\begin{aligned}
\frac{\theta}{2} E\left[\left(Y_{m}^{(k)}\right)^{i+2}\left(Y_{n}^{(k)}\right)^{j}\right]+\beta E\left[\left(Y_{m}^{(k)}\right)^{i+1}\left(Y_{n}^{(k)}\right)^{j}\right]-\lambda E\left[\left(Y_{m}^{(k)}\right)^{i}\left(Y_{n}^{(k)}\right)^{j}\right] \\
=\frac{m \alpha \theta}{i+2}\left[E\left[\left(Y_{m+1}^{(k)}\right)^{i+2}\left(Y_{n}^{(k)}\right)^{j}\right]-E\left[\left(Y_{m}^{(k)}\right)^{i+2}\left(Y_{n}^{(k)}\right)^{j}\right]\right] \\
+\frac{m \alpha \beta}{i+1}\left[E\left[\left(Y_{m+1}^{(k)}\right)^{i+1}\left(Y_{n}^{(k)}\right)^{j}\right]-E\left[\left(Y_{m}^{(k)}\right)^{i+1}\left(Y_{n}^{(k)}\right)^{j}\right],\right]
\end{aligned}
$$

arranging (3.9) we get the result as given in (3.8).

Now putting $n=m+1$ and noting that $E\left[\left(Y_{m}^{(k)}\right)^{i}\left(Y_{m}^{(k)}\right)^{j}\right]=E\left[\left(Y_{m}^{(k)}\right)^{i+j}\right]$, the recurrence relation given in (3.7) can be easily obtained on the same line of proof (3.8). 
Remark 3.1. If we put

i): $\quad \beta=\lambda=0$ in (3.9), we get the recurrence relations for product moment of generalized upper records from Weibull distribution

$$
E\left[\left(Y_{m+1}^{(k)}\right)^{i+2}\left(Y_{n}^{(k)}\right)^{j}\right]=\left(1+\frac{i+2}{2 \alpha m}\right) E\left[\left(Y_{m}^{(k)}\right)^{i+2}\left(Y_{n}^{(k)}\right)^{j}\right]
$$

at $i^{\prime}=i+2$, we get similar result as obtained by Khan and Khan [18].

ii): $\quad \beta=\lambda=0$ and $\alpha=\frac{1}{2}$ in (3.9), we get the recurrence relations for product moment of generalized upper records from the exponential distribution

$$
E\left[\left(Y_{m+1}^{(k)}\right)^{i+2}\left(Y_{n}^{(k)}\right)^{j}\right]=\left(1+\frac{i+2}{m}\right) E\left[\left(Y_{m}^{(k)}\right)^{i+2}\left(Y_{n}^{(k)}\right)^{j}\right]
$$

at $i^{\prime}=i+2$, we get similar result as obtained by Khan and Khan [18].

iii): $\quad \beta=\lambda=0$ and $\alpha=1$ in (3.9), we get the recurrence relations for product moment of generalized upper records from Rayleigh distribution

$$
E\left[\left(Y_{m+1}^{(k)}\right)^{i+2}\left(Y_{n}^{(k)}\right)^{j}\right]=\left(1+\frac{i+2}{2 m}\right) E\left[\left(Y_{m}^{(k)}\right)^{i+2}\left(Y_{n}^{(k)}\right)^{j}\right]
$$

at $i^{\prime}=i+2$, we get similar result as obtained by Khan and Khan [18].

iv): $\lambda=0$ and $\alpha=1$ in (3.9), we get the recurrence relations for product moment of generalized upper records from the LED

$$
\begin{aligned}
E\left[\left(Y_{m+1}^{(k)}\right)^{i+2}\left(Y_{n}^{(k)}\right)^{j}\right]= & \left(1+\frac{i+2}{2 m}\right) E\left[\left(Y_{m}^{(k)}\right)^{i+2}\left(Y_{n}^{(k)}\right)^{j}\right] \\
& +\frac{(i+2) \beta}{(i+1) \theta}\left[\left(1+\frac{i+1}{m}\right) E\left[\left(Y_{m}^{(k)}\right)^{i+1}\left(Y_{n}^{(k)}\right)^{j}\right]-E\left[\left(Y_{m+1}^{(k)}\right)^{i+1}\left(Y_{n}^{(k)}\right)^{j}\right]\right] .
\end{aligned}
$$

v): Setting $\beta=0$ in (3.8) we get the recurrence relations for product moment of generalized upper records from the generalized Rayleigh distribution

$$
E\left[\left(Y_{m}^{(k)}\right)^{i}\left(Y_{n}^{(k)}\right)^{j}\right]=\frac{m \alpha \theta}{(i+2) \lambda}\left[\left(1+\frac{i+2}{2 m \alpha}\right) E\left[\left(Y_{m}^{(k)}\right)^{i+2}\left(Y_{n}^{(k)}\right)^{j}\right]-E\left[\left(Y_{m+1}^{(k)}\right)^{i+2}\left(Y_{n}^{(k)}\right)^{j}\right]\right]
$$

vi): Putting $j=0$ in (3.8) we get the recurrence relation for single moment of generalized record value from the GLED.

Corollary 3.1. The recurrence relation for product moments of the upper record values from GLED has the form

$$
\begin{aligned}
\lambda E\left[\left(X_{U_{m}}\right)^{i}\left(X_{U_{n}}\right)^{j}\right]= & \frac{m \alpha \theta}{i+2}\left[\left(1+\frac{i+2}{2 m \alpha}\right) E\left[\left(X_{U_{m}}\right)^{i+2}\left(X_{U_{n}}\right)^{j}\right]-E\left[\left(X_{U_{m+1}}\right)^{i_{+} 2}\left(X_{U_{n}}\right)^{j}\right]\right] \\
& +\frac{m \alpha \beta}{i+1}\left[\left(1+\frac{i+1}{m \alpha}\right) E\left[\left(X_{U_{m}}\right)^{i+1}\left(X_{U_{n}}\right)^{j}\right]-E\left[\left(X_{U_{m+1}}\right)^{i+1}\left(X_{U_{n}}\right)^{j}\right]\right] .
\end{aligned}
$$

Remark 3.2. If we put

i): $\quad \beta=\lambda=0$ in (3.10), we get the recurrence relation of upper records from Weibull distribution, which is similar to the result as given by Balakrishnan and Chan [25] for $i^{\prime}=i+2$.

ii): $\quad \beta=\lambda=0$ and $\alpha=\frac{1}{2}$ in (3.10), we get the recurrence relation of upper records from the exponential distribution, which is similar to the result as given by Balakrishnan and Chan [25] for $i^{\prime}=i+2$.

iii): $\quad \beta=\lambda=0$ and $\alpha=1$ in (3.10), we get the recurrence relation of upper records from Rayleigh distribution, which is similar to the result as given by Balakrishnan and Chan [25] for $i^{\prime}=i+2$. 
iv): $\lambda=0$ and $\alpha=1$ in (3.10), we get the recurrence relations for upper records from the LED

$$
\begin{aligned}
E\left[\left(X_{U_{m+1}}\right)^{i+2}\left(X_{U_{n}}\right)^{j}\right]= & \left(1+\frac{i+2}{2 m}\right) E\left[\left(X_{U_{m}}\right)^{i+2}\left(X_{U_{n}}\right)^{j}\right] \\
& +\frac{(i+2) \beta}{(i+1) \theta}\left[\left(1+\frac{i+1}{m}\right) E\left[\left(X_{U_{m}}\right)^{i+i}\left(X_{U_{n}}\right)^{j}\right]-E\left[\left(X_{U_{m+1}}\right)^{i+1}\left(X_{U_{n}}\right)^{j}\right]\right] .
\end{aligned}
$$

v): Setting $\beta=0$ in (3.10) we get the recurrence relations of upper records from the generalized Rayleigh distribution

$$
\begin{aligned}
& E\left[\left(X_{U_{m}}\right)^{i}\left(X_{U_{n}}\right)^{j}\right] \\
& =\frac{m \alpha \theta}{(i+2) \lambda}\left[\left(1+\frac{i+2}{2 m \alpha}\right) E\left[\left(X_{U_{m}}\right)^{i+2}\left(X_{U_{n}}\right)^{j}\right]-E\left[\left(X_{U_{m+1}}\right)^{i+2}\left(X_{U_{n}}\right)^{j}\right]\right] .
\end{aligned}
$$

vi): Putting $j=0$ in (3.10) we get (2.12), the recurrence relation for single moment of upper record value from the GLED.

\section{CHARACTERIZATION}

This section contains the characterizations of GLED, we start with the following result of Lin [26].

PROPOSITION. Let $n_{0}$ be any fixed non-negative integer, $-\infty \leq a<b \leq \infty$ and $g(x) \geq 0$ an absolutely continuous function with $g^{\prime}(x) \neq 0$ a.e. on $(a, b)$. Then the sequence of functions $\left\{(g(x))^{n} e^{-g(x)}, n \geq n_{0}\right\}$ is complete in $\mathrm{L}(a, b)$ if $g(x)$ is strictly monotone on $(a, b)$.

Theorem 4.1. Fix a positive integer $k \geq 1$ and let $j$ be any non-negative integer. A necessary and sufficient condition for a random variable $X$ to be distributed with $p d f$ given by (1.1) is that

$$
\begin{aligned}
E\left(Y_{n}^{(k)}\right)^{j}= & \frac{n \alpha \theta}{(j+2) \lambda}\left[\left(1+\frac{j+2}{2 n \alpha}\right) E\left(Y_{n}^{(k)}\right)^{j+2}-E\left(Y_{n+1}^{(k)}\right)^{j+2}\right] \\
& +\frac{n \alpha \beta}{(j+1) \lambda}\left[\left(1+\frac{j+1}{n \alpha}\right) E\left(Y_{n}^{(k)}\right)^{j+1}-E\left(Y_{n+1}^{(k)}\right)^{j+1}\right]
\end{aligned}
$$

for $n=1,2, \ldots$

Proof. The necessary part follows from (2.9). On the other hand if the recurrence relation (4.1) is satisfied, then on rearranging (4.1)

$$
\begin{aligned}
& \frac{\theta}{2}\left[E\left(Y_{n}^{(k)}\right)^{j+2}\right]+\beta\left[E\left(Y_{n}^{(k)}\right)^{j+1}\right]-\lambda\left[E\left(Y_{n}^{(k)}\right)^{j}\right] \\
& =\frac{n \alpha \theta}{j+2}\left[E\left(Y_{n+1}^{(k)}\right)^{j+2}-E\left(Y_{n}^{(k)}\right)^{j+2}\right]+\frac{n \alpha \beta}{j+1}\left[E\left(Y_{n+1}^{(k)}\right)^{j+1}-E\left(Y_{n}^{(k)}\right)^{j+1}\right]
\end{aligned}
$$

in view of Khan et al. [19], we have

$$
\begin{aligned}
\frac{k^{n}}{(n-1) !} \int_{\Psi}^{\infty}\left(\frac{\theta}{2} x^{2}+\beta x-\lambda\right) x^{j}[-\ln \bar{F}(x)]^{n-1}[\bar{F}(x)]^{k-1} f(x) d x \\
=\frac{n \alpha \theta}{j+2}\left[\frac{(j+2) k^{n}}{n !} \int_{\Psi}^{\infty} x^{j+1}[-\ln \bar{F}(x)]^{n}[\bar{F}(x)]^{k} d x\right] \\
+\frac{n \alpha \beta}{j+1}\left[\frac{(j+1) k^{n}}{n !} \int_{\Psi}^{\infty} x^{j}[-\ln \bar{F}(x)]^{n}[\bar{F}(x)]^{k} d x\right],
\end{aligned}
$$

which implies

$$
\begin{aligned}
& \frac{k^{n}}{(n-1) !} \int_{\Psi}^{\infty} x^{j}[-\ln \bar{F}(x)]^{n-1}[\bar{F}(x)]^{k-1} \\
& \times\left\{\left(\frac{\theta}{2} x^{2}+\beta x-\lambda\right) f(x)-\alpha(\theta x+\beta)[-\ln \bar{F}(x)][\bar{F}(x)]\right\} d x=0
\end{aligned} .
$$


Now follow from the above proposition, we get

$$
\left(\frac{\theta}{2} x^{2}+\beta x-\lambda\right) f(x)=\alpha(\beta+\theta x)[-\ln \bar{F}(x)][\bar{F}(x)]
$$

which proves that $f(x)$ has the form as given in (1.3).

Theorem 4.2. For a positive integer $k, i$ and $j$ be a non-negative integer, a necessary and sufficient condition for a random variable $X$ to be distributed with $p d f$ given by (1.1) and for $1 \leq m \leq n-2$, is that

$$
\begin{aligned}
E\left[\left(Y_{m}^{(k)}\right)^{i}\left(Y_{n}^{(k)}\right)^{j}\right]= & \frac{m \alpha \theta}{(i+2) \lambda}\left[\left(1+\frac{i+2}{2 m \alpha}\right) E\left[\left(Y_{m}^{(k)}\right)^{i+2}\left(Y_{n}^{(k)}\right)^{j}\right]-E\left[\left(Y_{m+1}^{(k)}\right)^{i+2}\left(Y_{n}^{(k)}\right)^{j}\right]\right] \\
& +\frac{m \alpha \beta}{(i+1) \lambda}\left[\left(1+\frac{i+1}{m \alpha}\right) E\left[\left(Y_{m}^{(k)}\right)^{i+1}\left(Y_{n}^{(k)}\right)^{j}\right]-E\left[\left(Y_{m+1}^{(k)}\right)^{i+1}\left(Y_{n}^{(k)}\right)^{j}\right]\right] .
\end{aligned}
$$

Proof. The necessary part follows from (3.8). On the other hand if the relation in (4.2) is satisfied, then arranging the (4.2)

$$
\begin{gathered}
\frac{\theta}{2} E\left[\left(Y_{m}^{(k)}\right)^{i+2}\left(Y_{n}^{(k)}\right)^{j}\right]+\beta E\left[\left(Y_{m}^{(k)}\right)^{i+1}\left(Y_{n}^{(k)}\right)^{j}\right]-\lambda E\left[\left(Y_{m}^{(k)}\right)^{i}\left(Y_{n}^{(k)}\right)^{j}\right] \\
=\frac{m \alpha \theta}{i+2}\left[E\left[\left(Y_{m+1}^{(k)}\right)^{i+2}\left(Y_{n}^{(k)}\right)^{j}\right]-E\left[\left(Y_{m}^{(k)}\right)^{i+2}\left(Y_{n}^{(k)}\right)^{j}\right]\right] \\
+\frac{m \alpha \beta}{i+1}\left[E\left[\left(Y_{m+1}^{(k)}\right)^{i+1}\left(Y_{n}^{(k)}\right)^{j}\right]-E\left[\left(Y_{m}^{(k)}\right)^{i+1}\left(Y_{n}^{(k)}\right)^{j}\right]\right] .
\end{gathered}
$$

On using Lemma 3.1, we have

$$
\begin{aligned}
& \frac{k^{n}}{(m-1) !(n-m-1) !} \int_{\Psi}^{\infty} \int_{\Psi}^{y}\left(\frac{\theta}{2} x^{2}+\beta x-\lambda\right) x^{i} y^{j}[-\ln \bar{F}(x)]^{m-1} \frac{f(x)}{\bar{F}(x)} \\
& \times[\ln \bar{F}(x)-\ln \bar{F}(y)]^{n-m-1}[\bar{F}(y)]^{k-1} f(y) d x d y \\
= & \frac{\alpha \theta k^{n}}{(m-1) !(n-m-1) !} \times \int_{\Psi}^{\infty} \int_{\Psi}^{y} x^{i+1} y^{j}[-\ln \bar{F}(x)]^{m}[\ln \bar{F}(x)-\ln \bar{F}(y)]^{n-m-1}[\bar{F}(y)]^{k-1} f(y) d x d y \\
& +\frac{\alpha \beta k^{n}}{(m-1) !(n-m-1) !}+\int_{\Psi}^{\infty} \int_{\Psi}^{y} x^{i} y^{j}[-\ln \bar{F}(x)]^{m}[\ln \bar{F}(x)-\ln \bar{F}(y)]^{n-m-1}[\bar{F}(y)]^{k-1} f(y) d x d y .
\end{aligned}
$$

which implies

$$
\begin{aligned}
& \int_{\Psi}^{\infty} \int_{\Psi}^{y} x^{i} y^{j}[-\ln \bar{F}(x)]^{m-1}[\ln \bar{F}(x)-\ln \bar{F}(y)]^{n-m-1}[\bar{F}(y)]^{k-1} f(y) \\
& \times\left\{\left(\frac{\theta}{2} x^{2}+\beta x-\lambda\right) \frac{f(x)}{[\bar{F}(x)]}-\alpha(\theta x+\beta)[-\ln \bar{F}(x)]\right\} d x d y=0 .
\end{aligned}
$$

Now, follow from the above proposition with

$$
\left(\frac{\theta}{2} x^{2}+\beta x-\lambda\right) f(x)=\alpha(\beta+\theta x)[-\ln \bar{F}(x)][\bar{F}(x)]
$$

which proves that $f(x)$ has the form as given in (1.3). 
Theorem 4.3. Let $X$ be a non-negative random variable having an absolutely continuous $d f F(x)$ and $F(0)=0$ and $0 \leq F(x) \leq 1$ for all $x$ $>0$, then

$$
E\left[\xi\left(Y_{n}^{(k)}\right) \mid Y_{m}^{(k)}=x\right]=\xi(x)\left(\frac{k}{k+1}\right)^{n-m}
$$

if and only if

$$
\bar{F}(x)=\exp \left\{-\left(\frac{\theta}{2} x^{2}+\beta x-\lambda\right)^{\alpha}\right\}, \quad x>\Psi
$$

where

$$
\xi(t)=\exp \left\{-\left(\frac{\theta}{2} t^{2}+\beta t-\lambda\right)^{\alpha}\right\}, \quad t>\Psi
$$

Proof. From (1.6), we have

$$
\begin{aligned}
E\left[\xi\left(Y_{n}^{(k)}\right) \mid Y_{m}^{(k)}=x\right]= & \frac{k^{n-m}}{(n-m-1) !} \\
& \times \int_{x}^{\infty} \xi(y)[\ln \bar{F}(x)-\ln \bar{F}(y)]^{n-m-1}\left[\frac{\bar{F}(y)}{\bar{F}(x)}\right]^{k-1} \frac{f(y)}{\bar{F}(x)} d y .
\end{aligned}
$$

By setting $u=\frac{\bar{F}(y)}{\bar{F}(x)}=\frac{\exp \left\{-\left(\frac{\theta}{2} y^{2}+\beta y-\lambda\right)^{\alpha}\right\}}{\exp \left\{-\left(\frac{\theta}{2} x^{2}+\beta x-\lambda\right)^{\alpha}\right\}}$ from (1.2) in (4.4), we have

$$
E\left[\xi\left(Y_{n}^{(k)}\right) \mid\left(Y_{m}^{(k)}\right)=x\right]=\frac{k^{n-m}}{(n-m-1) !} \xi(x) \int_{0}^{1} u^{k}[-\ln u]^{n-m-1} d u
$$

Therefore we have (see Gradshteyn and Ryzhik [27], p. 551)

$$
\int_{0}^{1}[-\ln x]^{\mu-1} x^{\nu-1} d x=\frac{\Gamma \mu}{v^{\mu}}, \quad \mu>0, \nu>0
$$

On using (4.6) in (4.5), we have the result given in (4.3).

To prove sufficient part, we have

$$
\begin{aligned}
\frac{k^{n-m}}{(n-m-1) !} \int_{x}^{\infty} \xi(y)[ & -\ln \bar{F}(y)+\ln \bar{F}(x)]^{n-m-1}[\bar{F}(y)]^{k-1} f(y) d y \\
= & {[\bar{F}(x)]^{k} g_{n \mid m}(x), }
\end{aligned}
$$

where

$$
g_{n \mid m}(x)=\xi(x)\left(\frac{k}{k+1}\right)^{n-m}
$$

Differentiating (4.7) both sides with respect to $x$, we get

$$
\begin{aligned}
& -\frac{k^{n-m}}{(n-m-2) !} \frac{f(x)}{\bar{F}(x)} \int_{x}^{\infty} \xi(y)[-\ln \bar{F}(y)+\ln \bar{F}(x)]^{n-m-2}[\bar{F}(y)]^{k-1} f(y) d y \\
& =g_{n \mid m}^{\prime}(x)[\bar{F}(x)]^{k}-k g_{n \mid m}(x)[\bar{F}(x)]^{k-1} f(x)
\end{aligned}
$$


or

$$
-k g_{n \mid m+1}(x)[\bar{F}(x)]^{k-1} f(x)=g_{n \mid m}^{\prime}(x)[\bar{F}(x)]^{k}-k g_{n \mid m}(x)[\bar{F}(x)]^{k-1} f(x) .
$$

Therefore,

$$
\frac{f(x)}{\bar{F}(x)}=-\frac{g_{n \mid m}^{\prime}(x)}{k\left[g_{n \mid m+1}(x)-g_{n \mid m}(x)\right]}=-\frac{\xi^{\prime}(x)}{\xi(x)},
$$

where

$$
\begin{aligned}
& g_{n \mid m}^{\prime}(x)=\xi^{\prime}(x)\left(\frac{k}{k+1}\right)^{n-m}, \\
& g_{n \mid m+1}(x)-g_{n \mid m}(x)=\frac{1}{k} \xi(x)\left(\frac{k}{k+1}\right)^{n-m},
\end{aligned}
$$

Integrating both the sides (4.8) with respect to $x$ between $(\Psi, y)$, which gives $F^{-}(x)=\xi(x)$, the sufficiency part is proved.

\section{CONCLUSION}

In this study, we demonstrate the explicit expression as well as recurrence relation for the moments of $k$-th upper record values from the GLED. These relations can be used to reduce the amount of direct computation and moments of any order can be calculated easily. To verify the designed models which is required in probability distribution, we used the results of the characterization. At the different values of parameters, we reduced some well-known results. We can explore our study for generalized order statistics which contains several models of order random variates.

\section{CONFLICTS OF INTEREST}

The authors are declare no competing interests.

\section{AUTHORS' CONTRIBUTIONS}

Mahfooz Alam proposed the concept the paper and calculate the existing results. M.A. Khan contributed in writing-original draft preparation. R.U. Khan performed revision and improve the quality of the draft. All authors have read and agreed to the published version of the manuscript.

\section{Funding Statement}

This research received no external funding.

\section{ACKNOWLEDGMENTS}

The authors acknowledge with thanks the concrete suggestions and comments of the learned Referees, which led to an overall improvement of the paper. The authors are also grateful to Dr. Z. Akhter, Department of Statistics, University of Delhi for his help and suggestions throughout the preparation of this paper.

\section{REFERENCES}

1. M.A.W. Mahmoud, F.M.A. Alam, Stat. Probab. Lett. 80 (2010), 1005-1014.

2. C.S. Lee, H.J. Tsai, Stat. Probab. Lett. 124 (2017), 49-54.

3. K.N. Chandler, J. R. Stat. Soc. 14 (1952), 220-228.

4. M. Ahsanullah, Record Statistics, Nova Science Publishers, New York, NY, USA, 1995.

5. U. Kamps, A Concept of Generalized Order Statistics, B.G. Teubner, Stuttgart, Germany, 1995.

6. B.C. Arnold, N. Balakrishnan, H.N. Nagaraja, Records, Wiley, New York, NY, USA, 1998.

7. M. Ahsanullah, V.B. Nevzorov, Record via Probability Theory, Atlantis Press, Paris, France, 2015.

8. W. Dziubdziela, B. Kopcoiński, Appl. Math. (Warsaw). 15 (1976), 187-190. 
9. S. Minimol, P. Thomos, Braz. J. Probab. Stat. 27 (2013), 487-501.

10. V.B. Nevzorov, Records: Mathematical Theory, Translation of Mathematical Monographs, vol. 194, American Mathematical Society, Providence, RI, USA, 2001.

11. Z. Grudzień, Characterization of Distribution of Time Limits in Record Statistics as Well as Distributions and Moments of Linear Record Statistics from the Samples of Random Numbers, Praca Doktorska, UMCS, Lublin, Poland, 1982.

12. Z. Grudzień, D. Szynal, Prob. Stat. Decis. Theory A. (1983), 119-127.

13. Z. Grudzień, D. Szynal, J. Appl. Stat. Sci. 5 (1997), 259-266.

14. P. Pawlas, D. Szynal, J. Appl. Stat. Sci. 7 (1998), 53-62.

15. P. Pawlas, D. Szynal, Commun. Stat. Theory Methods. 28 (1999), 1699-1709.

16. P. Pawlas, D. Szynal, J. Appl. Stat. Sci. 10 (2000), 17-26.

17. S. Minimol, P. Thomos, J. Appl. Stat. Sci. 10 (2014), 1726.

18. M.A. Khan, R.U. Khan, Int. J. Comput. Theory. Stat. 3 (2016), 75-80.

19. R.U. Khan, M.A. Khan, M.A.R. Khan, Stat. Optim. Inf. Comput. 5 (2017), 127-136.

20. R.U. Khan, M.A. Khan, J. Egyptian Math. Soc. 23 (2015), 558-562.

21. B. Singh, R.U. Khan, M.A.R. Khan, Thail. Stat. 17 (2019), 93-103.

22. H.N. Nagaraja, Aust. J. Stat. 20 (1978), 176-182.

23. M. Ahsanullah, Pak. J. Stat. 3A (1987), 17-40.

24. M. Ahsanullah, Int. J. Stat. Sci. 11 (2011), 111-123.

25. N. Balakrishnan, P.S. Chan, Nat. Inst. Standard Technol. J. Res. Special Publ. 899 (1993), 41-51.

26. G.D. Lin, Tôhoku Math. J. 38 (1986), 595-598.

27. I.S. Gradshteyn, I.M. Ryzhik, Tables of Integrals, Series of Products, Academic Press, New York, NY, USA, 2007. 